\title{
Apolipoprotein E Genotype in Very Preterm Neonates with Intrauterine Growth Restriction: An Analysis of the German Neonatal Network Cohort
}

\author{
Stephen Norda, ${ }^{1}$ Tanja K. Rausch, ${ }^{2}$ Thorsten Orlikowsky, ${ }^{3}$ Matthias Hütten, ${ }^{3}$ \\ Sören Schulz, ${ }^{4}$ Wolfgang Göpel, ${ }^{4}$ and Ulrich Pecks ${ }^{1,5}$ \\ ${ }^{1}$ Department of Obstetrics and Gynecology, University Hospital of the RWTH Aachen, Aachen, Germany \\ ${ }^{2}$ Department of Medical Biometrics and Statistics, University of Lübeck, Lübeck, Germany \\ ${ }^{3}$ Department of Neonatology, University Children's Hospital of the RWTH Aachen, Aachen, Germany \\ ${ }^{4}$ Department of Pediatrics, University Hospital UKSH Lübeck, Lübeck, Germany \\ ${ }^{5}$ Department of Obstetrics and Gynecology, University Hospital UKSH Kiel, Kiel, Germany
}

Correspondence should be addressed to Ulrich Pecks; upecks@ukaachen.de

Received 27 January 2017; Revised 8 March 2017; Accepted 23 March 2017; Published 5 April 2017

Academic Editor: Erich Cosmi

Copyright (c) 2017 Stephen Norda et al. This is an open access article distributed under the Creative Commons Attribution License, which permits unrestricted use, distribution, and reproduction in any medium, provided the original work is properly cited.

\begin{abstract}
Aim. Cord blood of intrauterine growth restricted (IUGR) neonates displays lipid changes towards atherosclerotic profiles. Apolipoprotein E (ApoE) and its isoforms (e2, e3, and e4) are involved in the regulation of lipid metabolism. Specifically, ApoE e4 has been associated with atherosclerotic diseases, while e2 has a favorable effect. We therefore hypothesized that ApoE e4 haplotype is frequently observed in IUGR neonates and contributes to impaired fetal growth and the association of IUGR with cardiovascular and metabolic diseases later in life. Methods. A cohort of 4885 preterm infants $(\geq 22+0$ and $<32+0$ weeks of gestation and birth weight below $1500 \mathrm{~g}$ ) from the GNN study cohort was analyzed. Neonates were categorized into subgroups of $<3 \mathrm{rd}$, 3rd-10th, and $>10$ th birth weight percentile. Analysis of the single nucleotides rs429358 and rs7412, identifying the ApoE genotype, was carried out using TaqMan ${ }^{\circledR}$ SNP genotyping assays. The proportional odds model was used to assess data. Results. No association was found between genotype and birth weight percentiles in each of the subgroups. Conclusion. ApoE genotype and low birth weight depict two distinct risk factors for cardiovascular disease without being directly associated.
\end{abstract}

\section{Introduction}

Inadequate birth weight, compared to population based standards, has a complex pathophysiology not yet entirely understood. Disrupted placentation and consequently insufficient placental vascular supply of nutrients and oxygen to the fetus, among other factors, are believed to be involved in intrauterine growth restriction (IUGR) and to result in small for gestational age (SGA) neonates $[1,2]$. As such it shares common pathomechanisms with preeclampsia. Moreover, preeclampsia and IUGR often occur simultaneously especially if the onset is early preterm $[3,4]$. Hence, both diseases have been described as "placental syndrome." The clinical outcome of IUGR born babies is worse compared to neonates born with normal weight. Restricted fetal growth is believed to increase the risk for adverse neurophysiologic development, cardiovascular disease, and dyslipidemia in later life [5-9]. Atherogenic fetal serum lipid configurations have been associated with IUGR by previous research. IUGR born babies were found to have lower concentrations of highdensity lipoprotein cholesterol (HDL-C), known for having an anti-inflammatory effect and protective properties against the development of atherosclerosis, while triglycerides and oxidized low-density lipoprotein (oxLDL) levels were elevated in samples of umbilical blood compared to adequate weight newborns $[5,10,11]$. The results propose that disrupted cholesterol and triglyceride handling plays a role in causing suboptimal fetal development and exposes the newborn to an atherosclerotic environment early, consequentially giving raise to irreversible damage to vessels [6]. 
Apolipoprotein E (ApoE) is an important circulating serum protein involved in transporting lipids and cholesterol and regulating lipid levels. Its regulatory functions have been attributed to many biophysiological processes including neuronal growth and modulation of oxidant and inflammatory processes $[12,13]$. The ApoE gene has three allelic variants (e2, e3, and e4). The three haplotypes are determined by the SNPs rs429358 and rs7412. Variations in these nucleotides are determined by nucleobases T-T (e2), T-C (e3), and C-C $(\mathrm{e} 4)[14,15]$. Despite their minor structural disparities, the three variants e2, e3, and e4 and their corresponding amino acid polymorphisms exhibit differences in individual binding properties to different receptors and display diverse effects on lipoprotein metabolism [12].

The association of ApoE e4 with the development of dyslipidemia and cardiovascular disease has been described for the past two decades and is now well established [16, 17]. Recent studies provided evidence of isoform e 4 being present in individuals afflicted with severe cerebral palsy, promoting the development of Alzheimer's disease, and promoting the development of epilepsy. On the contrary, possibly due to its diverse binding properties, isoform e 2 displays a protective effect $[13,18,19]$. In newborns, carrying the ApoE e2 allele has been associated with lower fetal cord blood LDL-C levels and higher levels of HDL-C suggesting a beneficial effect of this genotype on blood lipid configuration [20,21].

Given the significant impact of ApoE genotype on serum lipid levels and the association of IUGR with altered lipid metabolism, we hypothesized fetal ApoE genotype to be a modulator of fetal growth and severity of IUGR. A link between ApoE genotype and birth weight percentile would prove the role of the APOE gene as modulator of fetal growth and consequently provide an explanation for the fact that impaired fetal development depicts a cardiovascular risk factor. Hence, the aim of the present study was to identify ApoE genotypes in IUGR neonates. We took advantage of a nation-wide genomic study of the German Neonatal Network (GNN) including more than 18000 preterm neonates born before 32 weeks of gestation or with very low birth weight below $1500 \mathrm{~g}$. The neonates were clustered according to birth weight percentiles with the lowest percentiles likely reflecting impaired fetal development. However, IUGR is not uniquely defined and neonates can be born small for their gestational age (SGA) by genetic determination and in the absence of a pathologic process $[5,22]$. Hence, in this study different SGA subtypes and scenarios have been considered including different cut-offs of percentiles to define SGA as well as factors associated with IUGR like hypertensive disorders in pregnancy. Identifying ApoE genotypes as an individual risk factor of IUGR or severity of IUGR has the potential to allow for better individual prenatal observation and postnatal treatment especially in preterm born neonates in need of intensive neonatal care.

\section{Methods}

2.1. Study Cohort. Subjects were enrolled in the GNN cohort between January 2009 and December 2015 by 54 currently participating neonatal intensive care units in Germany.
Preterm infants with a birth weight below $1500 \mathrm{~g}$ and born $<37+0$ weeks of gestation were enrolled. The GNN study was approved by the local committee for research in human subjects of the University of Lübeck (Germany), as well as by all of the local committees of participating centers. Written informed consent was obtained from parents with neonates eligible for the research and publication of data. A predefined clinical data set form, containing obstetric and neonatal data, was filled out by the attending physician. Data were then sent to the GNN coordinating center in Lübeck. Parental refusal, early neonatal death, and parents not asked to participate were reasons for nonenrollment. A specialized neonatologist surveyed the data quality by annual visits to the participating centers. Obstetrical and neonatal data collected included maternal origin, maternal age, gestational age, birth weight, fetal sex, singleton versus multiple birth, fetal malformations, presence of pathologic Doppler/IUGR, presence of maternal pregnancy induced hypertension (PIH) or preeclampsia, and presence of HELLP syndrome. Birth weight percentiles were calculated according to Voigt et al. [23]. Ultrasound measurements executed between 8 and 12 weeks of gestation determined fetal age.

2.2. Genotyping. ApoE genotyping was performed on buccal swabs and/or cord tissue transferred to the study center (University of Lübeck) together with the clinical data set form. DNA extraction was carried out by using commercial DNA purification kits (Qiagen, Hilden, Germany). The samples underwent real-time PCR. Genotyping of the APOE rs429358 and rs7412 single nucleotide polymorphisms (SNP), defining the e2, e3, and e4 allele, was performed by using TaqMan SNP Genotyping Assays (Applied Biosystems, Foster City, USA) according to the manufacturer's protocol. Genotyping of rs7412 and rs429358 was done in 10211 GNN-infants who were born between 2009 and 2014. Genotyping was successful in $93 \%$ of all patients for rs 429358 and $96 \%$ of all patients for rs7412 (90\% for both SNPs).

2.3. Patient Selection. We selected preterm infants with gestational age below $32+0$ weeks, a birth weight below 1500 grams, and European origin. Infants from multiple birth and with fetal malformations were excluded (Figure 1). To estimate interactions between ApoE genotype and abnormal fetal growth different scenarios were established: 4885 preterm neonates eligible for analysis were divided into three birth weight percentile groups: $>10$ th percentile $(n=3921)$, 3rd10th percentile $(n=625)$, and $<3$ rd percentile $(n=339)$. Those groups were further divided into subgroups according to antenatal clinical diagnosis of one of the subsequent features indicating a placental involvement (placental syndrome): (1) presence of maternal pregnancy induced hypertension (PIH including preeclampsia) and/or HELLP syndrome, (2) presence of IUGR and/or pathologic Doppler (maternal and/or fetal side) with/without PIH/HELLP, or (3) absence of criteria (1) or (2) (no placental syndrome). It was assumed that the subgroup with birth weight percentile $<3$ rd plus the clinical indication of IUGR/pathologic Doppler was most severely growth restricted while the subgroup of 


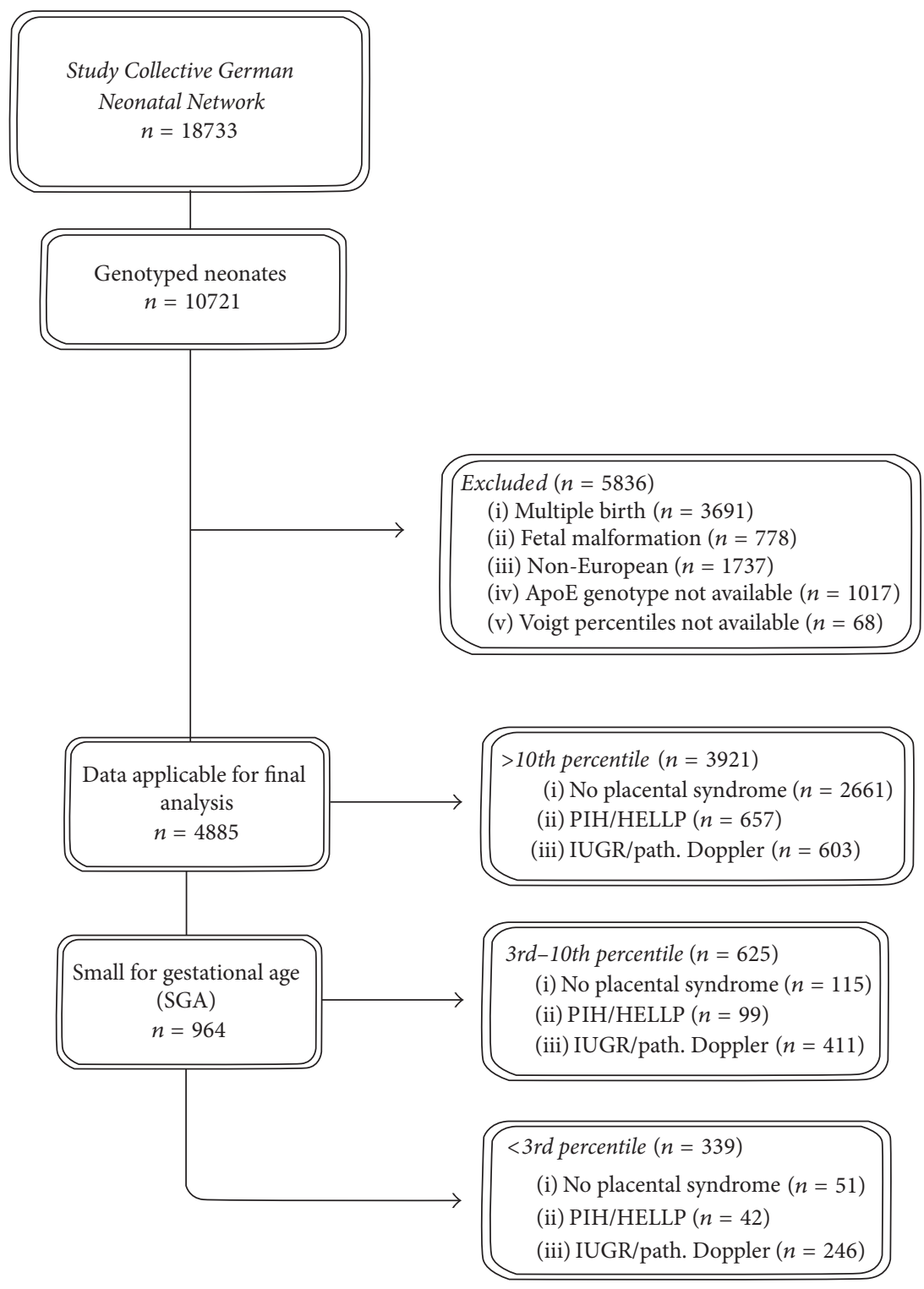

Figure 1: Inclusion/exclusion algorithm of probands of the German Neonatal Network. Multiple reasons for exclusion could apply. PIH includes preeclampsia. Patients with both "PIH/HELLP" and "IUGR/pathological Doppler ultrasound" were grouped into the "IUGR/pathological Doppler ultrasound" subgroup.

patients with birth weight percentile $>10$ th in the absence of (1) or (2) served as the most "pure" control group (Figure 2).

2.4. Statistical Analysis. Data analysis was performed using the SPSS 20.0 data analysis package (IBM, Munich, Germany). In order to evaluate the effect of the ApoE genotype on the birth weight in different SGA subtypes the proportional odds model was used to assess ordinal data. The model analyzes the likelihood to reach a higher ordinal level in relation to other variables. An equal slopes assumption of the data must be met which is tested beforehand. Our null hypothesis was $\left(H_{o}\right)=$ the ApoE genotype not affecting the Voigt birth weight percentile in different subgroups of premature IUGR. The type I error level was set to 0.05 . To correct for the multiple testing, nominal $p$ values were adjusted according to the Bonferroni-Holm procedure. Hardy Weinberg equilibrium for three alleles was assessed by chi-square test.

\section{Results}

Maternal and neonatal patient characteristics for each subgroup are shown in Table 1 . The average maternal age was 30.19 years $(\mathrm{SD} \pm 5.92)$. Gestational age on average was slightly smaller in the $>10$ th percentile weight group $(28,28 \pm$ 2.33 weeks) than in the $<3$ rd percentile weight group (29.92 \pm 4.25 weeks). Mean birth weight of $>10$ percentile group was $1075 \mathrm{~g}(\mathrm{SD} \pm 275 \mathrm{~g}), 924 \mathrm{~g}(\mathrm{SD} \pm 347 \mathrm{~g})$ in the $10 \mathrm{th}-3 \mathrm{rd}$, and $839 \mathrm{~g}(\mathrm{SD} \pm 421)$ in the $<3$ rd group. The percentage of female neonates varied from $47.4 \%$ to $50.4 \%$ among subgroups. From 
TABLE 1: Patient characteristics. Displayed values are based on data available.

\begin{tabular}{|c|c|c|c|c|c|c|}
\hline & \multicolumn{2}{|c|}{$>10 \mathrm{P}$} & \multicolumn{2}{|c|}{$3-10 \mathrm{P}$} & \multicolumn{2}{|c|}{$<3 \mathrm{P}$} \\
\hline & Mean & (SD) & Mean & $(\mathrm{SD})$ & Mean & $(\mathrm{SD})$ \\
\hline Maternal age (years) & 30.20 & $(5.90)$ & 30.04 & $(5.94)$ & 30.36 & $(6.05)$ \\
\hline $\begin{array}{l}\text { Gestational age at birth } \\
\text { (weeks) }\end{array}$ & 28.28 & $(2.33)$ & 29.74 & $(3.13)$ & 29.92 & $(4.25)$ \\
\hline \multirow[t]{2}{*}{ Birth weight (gram) } & 1075 & $(275)$ & 924 & $(347)$ & 839 & $(421)$ \\
\hline & frequency & $(\%)$ & frequency & $(\%)$ & frequency & $(\%)$ \\
\hline $\begin{array}{l}\text { Neonatal gender } \\
(n, \% \text { female })\end{array}$ & 1783 & $(47.4)$ & 593 & $(50.4)$ & 325 & $(48.3)$ \\
\hline
\end{tabular}

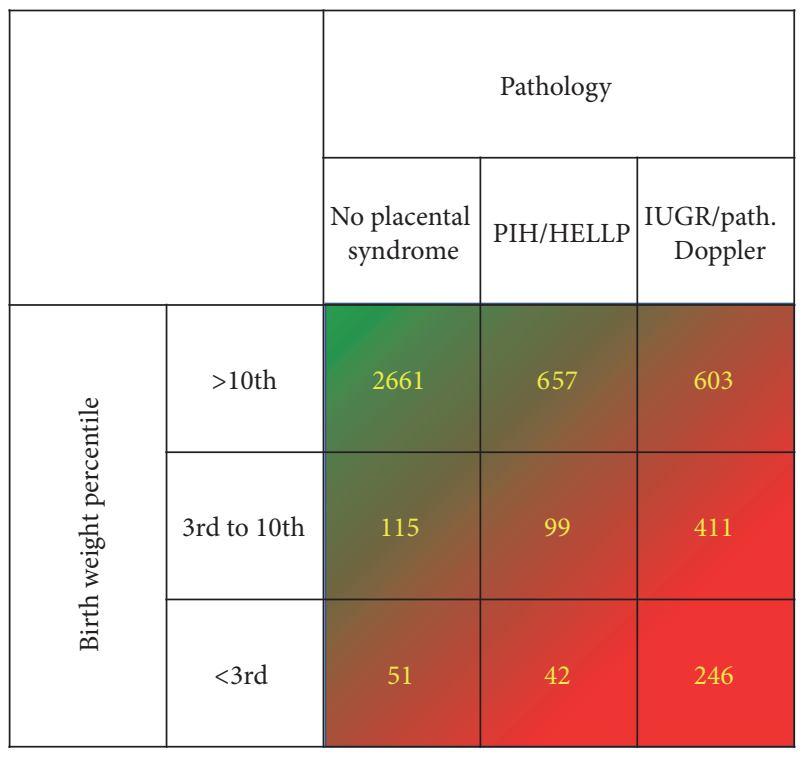

FIGURE 2: Frequencies of birth weight percentile groups and the presence or absence of PIH/HELLP syndrome or IUGR/pathological Doppler ultrasound as clinically indicated, of the total 4885 preterm neonates included into the analysis. Patients with both "PIH/HELLP" and "IUGR/pathological Doppler ultrasound" were grouped into the "IUGR/pathological Doppler ultrasound" subgroup.

the total group of 4885 probands, close to $95 \%$ of values were available for analysis of maternal and neonatal patient characteristics.

The frequencies of birth weight percentiles of neonates born without placental syndrome, in the presence of PIH/ HELLP or IUGR/pathological Doppler, respectively, are displayed in Figure 2.

Overall, haplotype frequencies were e2: 7,4\%, e3: 79\%, and e4: $13,6 \%$, respectively. ApoE genotype combinations found in our study population are shown in Table 2 . The genotypes showed a normal distributed Hardy Weinberg equilibrium. Genotype distribution per birth weight percentile and functional subgroup is depicted in Table 3.

Using proportional odds model and cumulative link model, respectively, interactive influence of ApoE genotypes and placental syndromes (three categories) on birth weight centiles have been estimated. As expected the groups
TABLE 2: Genotyping of the 4885 neonates.

\begin{tabular}{lcccccc}
\hline Genotype & e2/e2 & e3/e2 & e3/e3 & e3/e4 & e2/e4 & e4/e4 \\
\hline$n$ & 28 & 569 & 3049 & 1050 & 94 & 95 \\
$\%$ & 0.54 & 11.63 & 62.39 & 21.57 & 2.01 & 1.86 \\
\hline
\end{tabular}

PIH/HELLP and IUGR/pathological Doppler showed a statistically significant negative effect on birth weight percentile when compared to neonates born in absence of a placental syndrome (Table 4). None of the neonatal ApoE genotypes could be identified as having an influence on birth weight percentiles when e2/e 2 was used as a reference group.

To further investigate a possible correlation between ApoE genotype and birth weight percentile, the ApoE genotypes were pooled in groups containing the haplotype e2 (e2/e2 and e2/e3), haplotype e3 only, and haplotype e4 (e3/e4 and e4/e4). Thus the compiled allele groups did not show a significant effect on the birth weight percentiles in any of the analyzed subgroups compared to the groups e2/e2 and e2/e3 (data not shown).

\section{Discussion}

To our knowledge, the data set used in this study was the largest analyzed to date. 4885 preterm neonates were included in the analysis with the aim of identifying the effect of the ApoE genotype on proper fetal development. However, we did not find any association of ApoE haplotypes with birth weight percentiles independently of whether or not pregnancy was further complicated by a placental disorder characterized by PIH or IUGR.

Three prior studies investigated the ApoE genotype in IUGR/low birth weight children. Our results are in accordance with Akisu et al. who also found no correlation between the ApoE genotype and IUGR born newborns [24]. However, their results are not entirely applicable to the present study since all infants investigated had completed the 36 th week of gestation. Moreover, the study was massively underpowered as it included only 20 cases of IUGR. Another study by Szitanyi et al. retrospectively investigated the ApoE genotypes and birth weight of two groups of 10-11-year-old children. They hypothesized that both intrauterine undernutrition, demonstrated by a lower birth weight, and the ApoE 
TABLE 3: Genotype distribution (frequencies) of preterm neonates subgrouped by birth weight percentile and type of pregnancy related pathology. Patients with both, "PIH/HELLP" and "IUGR/pathological Doppler ultrasound," were grouped into the "IUGR/pathological Doppler ultrasound" subgroup.

\begin{tabular}{|c|c|c|c|c|c|c|c|}
\hline $\begin{array}{l}\text { Placental } \\
\text { syndrome }\end{array}$ & $\begin{array}{l}\text { Birth weight } \\
\text { percentile }\end{array}$ & $\mathrm{e} 2 / \mathrm{e} 2$ & $\mathrm{e} 3 / \mathrm{e} 2$ & e3/e3 & $\mathrm{e} 3 / \mathrm{e} 4$ & $\mathrm{e} 2 / \mathrm{e} 4$ & $\mathrm{e} 4 / \mathrm{e} 4$ \\
\hline \multirow{3}{*}{$\begin{array}{l}\text { No placental } \\
\text { syndrome }\end{array}$} & $>10$ & 15 & 321 & 1644 & 572 & 57 & 52 \\
\hline & $3-10$ & 1 & 17 & 70 & 22 & 3 & 2 \\
\hline & $<3$ & 1 & 8 & 28 & 12 & 1 & 1 \\
\hline \multirow{3}{*}{ PIH/HELLP } & $>10$ & 7 & 72 & 417 & 135 & 13 & 13 \\
\hline & $3-10$ & 0 & 10 & 61 & 26 & 1 & 1 \\
\hline & $<3$ & 0 & 2 & 29 & 9 & 0 & 2 \\
\hline \multirow{3}{*}{$\begin{array}{l}\text { IUGR/path. } \\
\text { Doppler }\end{array}$} & $>10$ & 1 & 63 & 399 & 117 & 9 & 14 \\
\hline & $3-10$ & 0 & 52 & 243 & 105 & 5 & 6 \\
\hline & $<3$ & 3 & 24 & 158 & 52 & 5 & 4 \\
\hline
\end{tabular}

TABLE 4: Analysis of interactive effect of ApoE genotype and subgroups with placental syndrome (PIH/HELLP, IUGR/pathologic Doppler) on birth weight percentile groups.

\begin{tabular}{|c|c|c|c|c|c|}
\hline Factor & Estimate & SE & $z$ value & Nominal $P$ & Adjusted P \\
\hline e2/e3 & 0.4891 & 0.5359 & 0.9127 & 0.36 & 1 \\
\hline $\mathrm{e} 3 / \mathrm{e} 3$ & 0.5667 & 0.5256 & 1.0781 & 0.28 & 1 \\
\hline $\mathrm{e} 3 / \mathrm{e} 4$ & 0.4305 & 0.5297 & 0.8128 & 0.42 & 1 \\
\hline $\mathrm{e} 4 / \mathrm{e} 4$ & 0.7221 & 0.6048 & 1.1939 & 0.23 & 1 \\
\hline $\mathrm{e} 2 / \mathrm{e} 4$ & 0.5635 & 0.6080 & 0.9268 & 0.35 & 1 \\
\hline PIH/HELLP & -1.2365 & 0.1224 & -10.1034 & $<0.0001$ & $<0.0001$ \\
\hline IUGR & -2.8398 & 0.0971 & -29.2359 & $<0.0001$ & $<0.0001$ \\
\hline
\end{tabular}

Results of analyzing the effect of individual ApoE genotypes with underlying maternal pathology, respectively, on birth weight percentile. Data were compared to reference group e2/e2 for genotype analysis and to no placental syndrome, respectively.

Patients with both, "PIH/HELLP" and "IUGR/pathological Doppler ultrasound," were grouped into the "IUGR/pathological Doppler ultrasound" subgroup. All values are rounded on four decimals. The type I error level was set to 0.05 . Adjusted $p$ values are adjusted according to the Bonferroni-Holm procedure.

genotype participate in the development of hypercholesterolemia in childhood [25]. Children were subdivided into tertiles with high and low levels of cholesterol, respectively. While the high-cholesterol group had significantly lower birth weight $(0.3 \mathrm{~kg})$ and ApoE e4 had a higher prevalence among this group, no association could be established between ApoE e4 and birth weight alone. Unfortunately, the study did not provide any data on weeks of gestation at birth, actual birth weight, birth weight percentiles, or the presence of IUGR/SGA in their study population of 139 children. Therefore, their results need to be addressed with caution. A third large study by Infante-Rivard et al. (449 newborns) using a family based study design consistently found a significantly reduced transmission of allele e2 to newborns affected with IUGR defined as birth weight below the 10th percentile. The authors concluded that allele e2 is protective against IUGR. However, their diagnosis of IUGR needs to be questioned, since birth weight percentile below the 10th percentile alone is not a reliable definition of IUGR. The authors themselves stated that exclusion of patients with histopathologically confirmed placental infarctions $(n=10)$ results into an even more statistically significant effect. This points to different pathomechanisms for specific IUGR/SGA subgroups [20].
Though we did not primarily aim to analyze the association between ApoE genotype and the incidence of preeclampsia and the HELLP syndrome, our study does partly allow us to draw certain conclusions. Both of the aforementioned diseases are pathologically closely related to IUGR. No association between the clinical diagnosis of pregnancy being terminated in the presence of a hypertensive disorder like preeclampsia or the HELLP syndrome has been observed in this study. The present study therefore contradicts a recent publication by Procopciuc et al. in which the fetal Apo e4 genotype was found to be an independent risk factor for preeclampsia compared to the other ApoE genotypes [26]. The diverging conclusions of their study are probably caused by the small sample-size $(n=141$ with 47 patients suffering on preeclampsia) with ApoE e4 not being normally distributed in their control group. Other authors did not find associations of ApoE genotype and the risk of preeclampsia when genotyping the mother [27-29] which corresponds with our observations in the fetus.

Despite these findings, there is increasing evidence that ApoE plays a pivotal role in fetal development and lipid metabolism. ApoE is the only apolipoprotein in the fetal circulation in concentrations as high as the mother's [30]. It is highly associated with fetal HDL-C that, in contrast to 
adults, is the predominant lipoprotein in the fetus at term [31]. In cord blood of fetuses with IUGR, both HDL-C and ApoE concentrations are largely reduced [32]. Interestingly, maternal ApoE genotype impacts fetal lipid concentration levels and vice versa. While the e 2 isoform in newborns is associated with elevated maternal LDL-C and apolipoprotein $\mathrm{B}$ (ApoB) levels, the same isoform in the mother is associated with lower LDL-C and ApoB levels and higher HDL-C levels in cord blood $[21,33]$. Maternal cholesterol in humans rises during pregnancy to guarantee fetal nutritional supply. Furthermore, cholesterol levels are affected by a number of factors, environmental and genetic [34]. One influencing candidate on cholesterol levels is therefore the APOE locus, either through its direct effects on the developing fetus, on the fetal lipoprotein metabolism, or through its effects on maternal cholesterol levels and the maternofetal lipoprotein metabolism. In IUGR fetal serum lipid levels resemble atherogenic profiles, with lower HDL-C concentrations and higher triglyceride levels as well as elevated ratios of oxidized LDL/LDL-C and LDL-C/HDL-C $[5,10,11]$. The relationship is believed to derive from the early susceptibility of tissue to damage while being in a state of plasticity $[6,7]$.

The physiologic effect of the ApoE genotype on lipoprotein levels and the development of atherosclerotic diseases are well established in humans. Though being born with low birth weight predisposes to cardiovascular disease later in life it is likely that the three haplotypes of the APOE gene and low birth weight depict two independent risk factors on cardiovascular disease, since our study does not suggest that the association between low birth weight and cardiovascular disease is due to ApoE genotype.

Our study is limited by two main aspects. First, preterm birth per se is a pathological condition; hence, our study lacks a valid control group of uncomplicated pregnancies. However, the overall distribution of ApoE genotypes was within the expected range for the German population. Second, the study may be biased due to the vague definition of IUGR in the absence of precise antenatal recorded criteria. Neonates with birth weights less than the 10th percentile of a population are SGA. The term SGA is descriptive and refers not to fetal growth velocity but to the birth weight of an infant. It does not reflect the causes that lead to the low birth weight. Most SGA neonates are born small by constitution and have constant growth patterns $[5,22,35]$. By contrast, IUGR indicates the presence of a pathophysiological process that slows down or inhibits fetal growth at a certain but usually unknown developmental stage during pregnancy. Hence, the diagnosis of IUGR is based ideally on the antenatal observation of a deceleration of fetal growth velocity by serial sonographic measurements showing a "crossing of percentiles" or other antenatal parameters of fetal well-being [22, 36]. Evidence is increasing that IUGR is a heterogeneous disorder that can be subdivided into clinical presentation of concomitant preeclampsia and early onset or late onset IUGR with a cutoff at 32 to 34 weeks of gestation [2,37]. Both, preeclampsia and increased resistance of the umbilical arteries (as indicated by resistance index/pulsatility index in Doppler sonography) are often associated with severe early onset IUGR. The indication of the presence of PIH, HELLP syndrome, IUGR, and antenatal pathological Doppler parameters in this study relies on rather clinical documentations that has not followed a strict study protocol and is not uniquely defined within the participating centers, since this was not the primary study aim of the GNN study. However, the inclusion criteria in this study are restricted to neonates born very preterm (early onset) making it a rather homogeneous study population. Moreover, the large size of our study population allowed us to investigate subgroups of very-low-birth-weight-infants according to various definitions of IUGR with high accuracy and low chance of error.

\section{Conclusion}

Genotyping our large cohort of 4885 preterm infants did not reveal an effect or dose-effect relationship of the fetal ApoE isoform on the birth weight percentile in all subgroups investigated. IUGR born infants display atherogenic blood lipid levels. Apolipoprotein $\mathrm{E}$ is known to regulate blood lipids and its three haplotypes are associated with distinct blood lipid patterns. Yet, the hypothesis of ApoE genotype affecting birth weight could be rejected since no direct effect was found. The stratification of maternal pathology in our large population size, that in earlier studies might have biased the results, could not alter this conclusion. Cholesterol levels and ApoE genotype depict major risk factors for CVD in later life. Our results, however, suggest that the ApoE genotype and low birth weight represent two synergistic factors exposing the fetus to an atherogenic environment and contributing to intrauterine programming without being directly associated to each other. The maternal genotype, known for affecting fetal blood lipids, could play a role and should be addressed in further studies.

\section{Conflicts of Interest}

The authors declare that they have no conflicts of interest.

\section{Acknowledgments}

The study has been done on behalf of the German Neonatal Network (GNN). The authors want to thank Rosa Papadantonaki and Tim Ewoldt for bringing the paper into its final form.

\section{References}

[1] J. H. W. Veerbeek, P. G. J. Nikkels, H. L. Torrance et al., "Placental pathology in early intrauterine growth restriction associated with maternal hypertension," Placenta, vol. 35, no. 9, pp. 696-701, 2014.

[2] S. Savchev, F. Figueras, M. Sanz-Cortes et al., "Evaluation of an optimal gestational age cut-off for the definition of early-and late-onset fetal growth restriction," Fetal Diagnosis and Therapy, vol. 36, no. 2, pp. 99-105, 2014.

[3] J. Villar, G. Carroli, D. Wojdyla et al., "Preeclampsia, gestational hypertension and intrauterine growth restriction, related or independent conditions?" American Journal of Obstetrics and Gynecology, vol. 194, no. 4, pp. 921-931, 2006. 
[4] U. Pecks, R. Caspers, B. Schiessl et al., "The evaluation of the oxidative state of low-density lipoproteins in intrauterine growth restriction and preeclampsia," Hypertension in Pregnancy, vol. 31, no. 1, pp. 156-165, 2012.

[5] U. Pecks, M. Brieger, B. Schiessl et al., "Maternal and fetal cord blood lipids in intrauterine growth restriction," Journal of Perinatal Medicine, vol. 40, no. 3, pp. 287-296, 2012.

[6] S. Visentin, F. Grumolato, G. B. Nardelli, B. Di Camillo, E. Grisan, and E. Cosmi, "Early origins of adult disease: low birth weight and vascular remodeling," Atherosclerosis, vol. 237, no. 2, pp. 391-399, 2014.

[7] D. J. P. Barker, C. Osmond, P. D. Winter, B. Margetts, and S. J. Simmonds, "Weight in infancy and death from ischaemic heart disease," The Lancet, vol. 334, no. 8663, pp. 577-580, 1989.

[8] M. El Ayoubi, J. Patkai, C. Bordarier et al., "Impact of fetal growth restriction on neurodevelopmental outcome at 2 years for extremely preterm infants: a single institution study," Developmental Medicine and Child Neurology, vol. 58, no. 12, pp. 12491256, 2016.

[9] S. Jarvis, S. V. Glinianaia, M.-G. Torrioli et al., "Cerebral palsy and intrauterine growth in single births: European collaborative study," The Lancet, vol. 362, no. 9390, pp. 1106-1111, 2003.

[10] J. A. D. Spencer, T. C. Chang, D. Crook et al., "Third trimester fetal growth and measures of carbohydrate and lipid metabolism in umbilical venous blood at term," Archives of Disease in Childhood: Fetal and Neonatal Edition, vol. 76, no. 1, pp. F21F25, 1997.

[11] L. Leduc, E. Delvin, A. Ouellet et al., "Oxidized low-density lipoproteins in cord blood from neonates with intra-uterine growth restriction," European Journal of Obstetrics Gynecology and Reproductive Biology, vol. 156, no. 1, pp. 46-49, 2011.

[12] J. Heeren, U. Beisiegel, and T. Grewal, "Apolipoprotein E recycling: implications for dyslipidemia and atherosclerosis," Arteriosclerosis, Thrombosis, and Vascular Biology, vol. 26, no. 3, pp. 442-448, 2006.

[13] E. Lien, G. Andersen, Y. Bao et al., "Genes determining the severity of cerebral palsy: the role of single nucleotide polymorphisms on the amount and structure of apolipoprotein e," Acta Paediatrica, vol. 104, no. 7, pp. 701-706, 2015.

[14] D. Seripa, G. D’Onofrio, F. Panza, L. Cascavilla, C. Masullo, and A. Pilotto, "The genetics of the human APOE polymorphism," Rejuvenation Research, vol. 14, no. 5, pp. 491-500, 2011.

[15] W. Koch, A. Ehrenhaft, K. Griesser et al., "TaqMan systems for genotyping of disease-related polymorphisms present in the gene encoding apolipoprotein E," Clinical Chemistry and Laboratory Medicine, vol. 40, no. 11, pp. 1123-1131, 2002.

[16] P. W. F. Wilson, M. G. Larson, R. H. Myers, P. A. Wolf, J. M. Ordovas, and E. J. Schaefer, "Apolipoprotein E alleles, dyslipidemia, and coronary heart disease: The Framingham Offspring Study," JAMA, vol. 272, no. 21, pp. 1666-1671, 1994.

[17] P. Perron, D. Brisson, M. Santuré et al., "Apolipoprotein E and lipoprotein lipase gene polymorphisms interaction on the atherogenic combined expression of hypertriglyceridemia and hyperapobetalipoproteinemia phenotypes," Journal of Endocrinological Investigation, vol. 30, no. 7, pp. 551-557, 2007.

[18] E. Lien, G. L. Andersen, Y. Bao et al., "Apolipoprotein E polymorphisms and severity of cerebral palsy: a cross-sectional study in 255 children in Norway," Developmental Medicine and Child Neurology, vol. 55, no. 4, pp. 372-377, 2013.

[19] J. E. Eichner, S. T. Dunn, G. Perveen, D. M. Thompson, K. E. Stewart, and B. C. Stroehla, "Apolipoprotein E polymorphism and cardiovascular disease: a HuGE review," American Journal of Epidemiology, vol. 155, no. 6, pp. 487-495, 2002.

[20] C. Infante-Rivard, E. Lévy, G.-E. Rivard, M. Guiguet, and J.-C. Feoli-Fonseca, "Small babies receive the cardiovascular protective apolipoprotein epsilon 2 allele less frequently than expected," Journal of Medical Genetics, vol. 40, no. 8, pp. 626629, 2003.

[21] O. S. Descamps, M. Bruniaux, P.-F. Guilmot, R. Tonglet, and F. R. Heller, "Lipoprotein concentrations in newborns are associated with allelic variations in their mothers," Atherosclerosis, vol. 172, no. 2, pp. 287-298, 2004.

[22] U. Pecks, I. Kirschner, M. Wölter et al., "Mass spectrometric profiling of cord blood serum proteomes to distinguish infants with intrauterine growth restriction from those who are small for gestational age and from control individuals," Translational Research, vol. 164, no. 1, pp. 57-69, 2014.

[23] M. Voigt, K. T. M. Schneider, H. Brinks et al., "11. Mitteilung: Unterschiede im somatischen Entwicklungsstand Neugeborener unter Berücksichtigung des Herkunftslandes der Mutter," Geburtshilfe und Frauenheilkunde, vol. 66, no. 4, pp. 391-399, 2006.

[24] M. Akisu, Z. Balim, H. Cetin et al., "The role of angiotensinconverting enzyme and apolipoprotein-E gene polymorphisms on lipid compositions in newborn infants with intrauterine growth restriction," Early Human Development, vol. 78, no. 2, pp. 95-103, 2004.

[25] P. Szitanyi, H. Pistulkova, J. A. Hubacek, H. Stuchlikova, and R. Poledne, "Apolipoprotein E/intrauterine undernutrition interaction and hypercholesterolemia in children," Physiological Research, vol. 57, no. 6, pp. 965-968, 2008.

[26] L. M. Procopciuc, G. Caracostea, G. Zaharie, and F. Stamatian, "Newborn APOE genotype influences maternal lipid profile and the severity of high-risk pregnancy-preeclampsia: interaction with maternal genotypes as a modulating risk factor in preeclampsia," Hypertension in Pregnancy, vol. 34, no. 3, pp. 271283, 2015.

[27] B. Jamalzei, S. Fallah, M. Kashanian, and M. Seifi, "Association of the apolipoprotein E variants with susceptibility to pregnancy with preeclampsia," Clinical Laboratory, vol. 59, no. 5-6, pp. 563570, 2013.

[28] J. Francoual, F. Audibert, P. Trioche et al., "Is a polymorphism of the apolipoprotein E gene associated with preeclampsia?" Hypertension in Pregnancy, vol. 21, no. 2, pp. 127-133, 2002.

[29] N. Makkonen, S. Heinonen, P. Kirkinen, M. Hiltunen, S. Helisalmi, and A. Mannermaa, "Apolipoprotein E alleles in women with pre-eclampsia," Journal of Clinical Pathology, vol. 54, no. 8, pp. 652-654, 2001.

[30] I. Sreckovic, R. Birner-Gruenberger, B. Obrist et al., "Distinct composition of human fetal HDL attenuates its anti-oxidative capacity," Biochimica et Biophysica Acta, vol. 1831, no. 4, pp. 737746, 2013.

[31] M. Augsten, H. Hackl, B. Ebner et al., "Fetal HDL/apoE: a novel regulator of gene expression in human placental endothelial Cells," Physiological Genomics, vol. 43, no. 22, pp. 1255-1262, 2011.

[32] U. Pecks, W. Rath, D. O. Bauerschlag et al., "Serum cholesterol acceptor capacity in intrauterine growth restricted fetuses," Journal of Perinatal Medicine, 2017.

[33] O. S. Descamps, M. Bruniaux, P.-F. Guilmot, R. Tonglet, and F. R. Heller, "Lipoprotein metabolism of pregnant women is associated with both their genetic polymorphisms and those of 
their newborn children," Journal of Lipid Research, vol. 46, no. 11, pp. 2405-2414, 2005.

[34] U. Pecks, W. Rath, N. Kleine-Eggebrecht et al., "Maternal serum lipid, estradiol, and progesterone levels in pregnancy, and the impact of placental and hepatic pathologies," Geburtshilfe und Frauenheilkunde, vol. 76, no. 7, pp. 799-808, 2016.

[35] A. Rosenberg, "The IUGR newborn," Seminars in Perinatology, vol. 32, no. 3, pp. 219-224, 2008.

[36] E. Cosmi, G. Ambrosini, D. D’Antona, C. Saccardi, and G. Mari, "Doppler, cardiotocography, and biophysical profile changes in growth-restricted fetuses," Obstetrics and Gynecology, vol. 106, no. 6, pp. 1240-1245, 2005.

[37] U. Pecks, W. Rath, N. Maass et al., "Fetal gender and gestational age differentially affect PCSK9 levels in intrauterine growth restriction," Lipids in Health and Disease, vol. 15, no. 1, p. 193, 2016. 


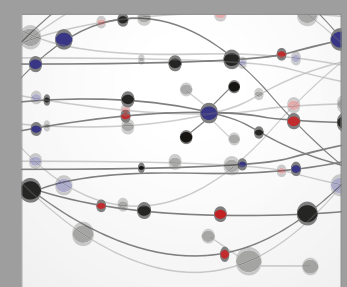

The Scientific World Journal
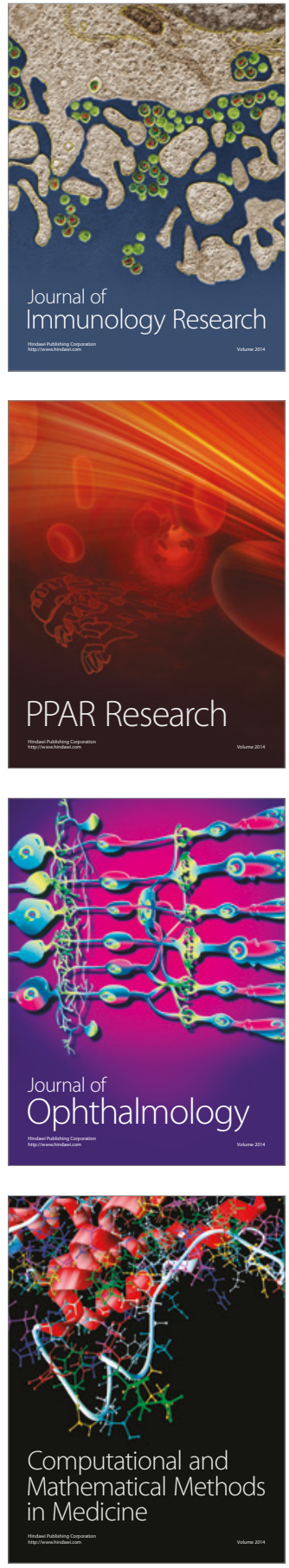

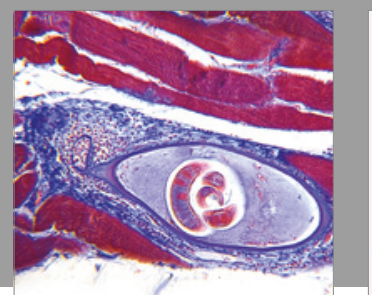

Gastroenterology Research and Practice
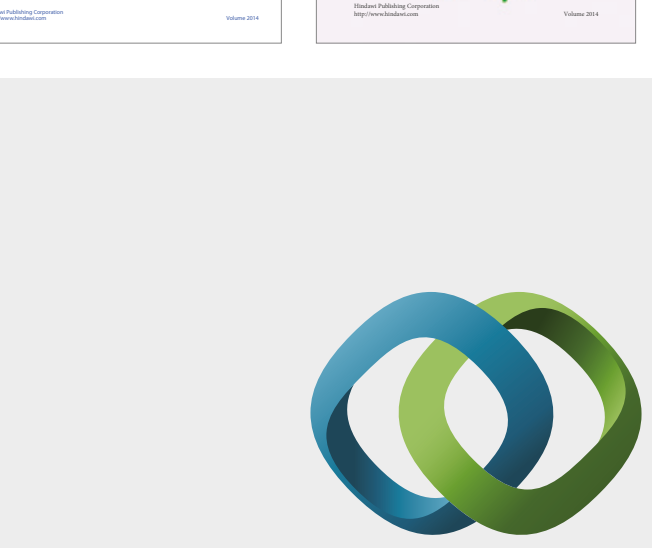

\section{Hindawi}

Submit your manuscripts at

https://www.hindawi.com
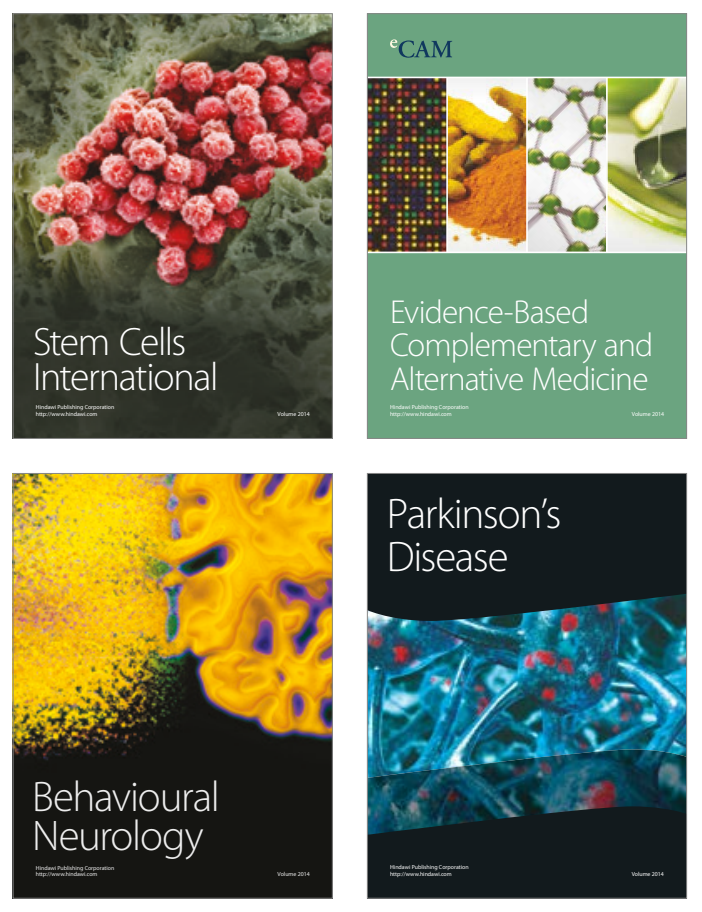
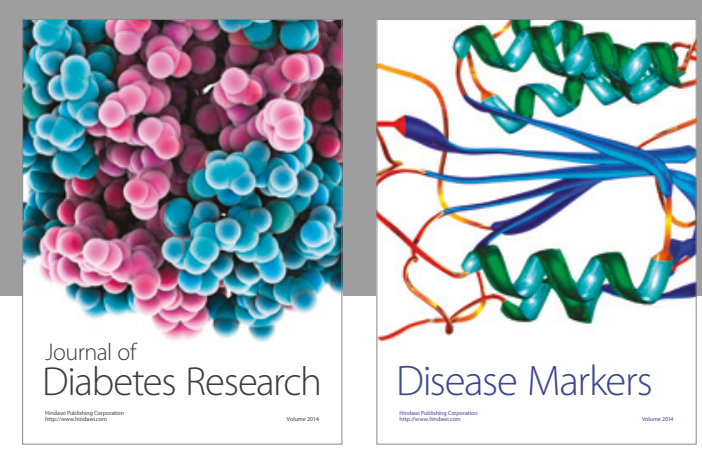

Disease Markers
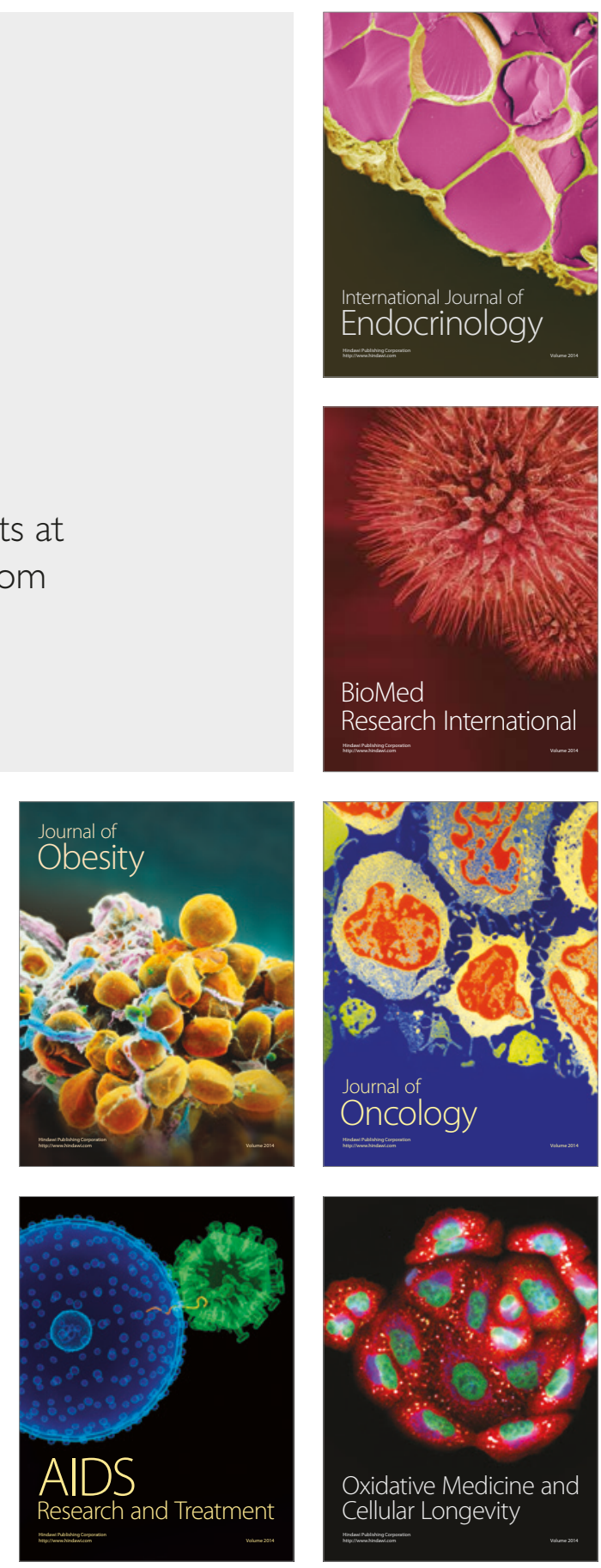